\title{
Intellectuels africains, patriotisme et panafricanisme : à propos de la fuite des cerveaux
}

\author{
Ernest-Marie Mbonda*
}

\section{Résumé}

Sous les apparences d'une simple description sociologique, la notion de fuite des cerveaux cache un jugement de valeur sur les devoirs des intellectuels à l'égard de leurs patries. On ne parlerait pas de « fuite » si l'on ne considérait pas tout intellectuel comme étant d'abord rattaché à un pays particulier, à un continent particulier, et ayant à son égard une certaine « obligation patriotique » de contribuer, de toute la force de ses capacités intellectuelles, à son développement, à sa prospérité et à son rayonnement. Les intellectuels qui ont choisi l'expatriation pourraient être accusés d'avoir voulu accorder la préférence à leurs intérêts particuliers, et de s'être désolidarisés de manière coupable du destin de leurs pays, de leur continent, de leur peuple, d'avoir manqué au devoir d'apporter une contribution, si modeste soit-elle, à la construction de sociétés africaines justes, démocratiques et prospères, et à la mise en œuvre des projets nationalistes et panafricanistes qui ont fleuri autour des indépendances. Mais peut-on considérer d'emblée le patriotisme (ainsi d'ailleurs que le nationalisme) comme une vertu et la fuite des cerveaux doit-elle être indexée en terme de vice ? N'est-il pas des situations où c'est la fuite des cerveaux elle-même qui devrait être considérée comme une vertu, au moins en ce qu'elle pourrait permettre de mettre au service de l'humanité entière et de sa propre société (à travers des États qui les reconnaissent et les valorisent) des talents qui autrement se seraient sclérosés ? Ces questions qui, en réalité contiennent implicitement notre hypothèse, constituent les lignes directrices de la réflexion que nous avons développée dans cet article.

\begin{abstract}
Under the guise of a simple sociological description, the concept of brain drain hides a value judgement on the duties of intellectuals towards their homelands. We would not talk about 'drain' if every intellectual was not primarily consid-
\end{abstract}

* Université catholique d'Afrique centrale, Faculté de philosophie, Yaoundé, Cameroun. 
ered as attached to a specific country, a specific continent, and as having some 'patriotic duty' to contribute, with all his/her intellectual capacity, to the development, prosperity and influence of their homeland. The intellectuals who have chosen emigration could be accused of prioritizing their interests, and shamefully dissociating themselves from the destiny of their countries, their continents and their peoples. Those intellectuals could be blamed for having failed to make a contribution, however modest it could be, to the construction of fair, democratic and prosperous African societies and to help in the implementation of those nationalist and pan-Africanist projects that were envisioned around the early post-independence period. Yet, is patriotism (as well as nationalism) to be considered as a virtue, and should brain drain be regarded as a vice? Aren't there situations where brain drain itself could be regarded as a virtue, at least in the sense that it could help to put at the service of humankind and its own society (through states that recognize and value it) talents that otherwise would have been left untapped? These are some of the questions, which in fact implicitly contain our assumption, guiding the reflection that we have developed in this article.

\section{Introdution}

Il y a quelques années, la Commission économique des Nations unies pour l'Afrique (cf. Programme-Cadre pour la mise en place, le renforcement et l'utilisation des capacités essentielles en Afrique, Addis-Abeba, 1996) fournissait à propos de la fuite des cerveaux les statistiques suivantes : entre 1960 et 1975, ce sont 27000 hauts cadres africains qui ont quitté leur continent pour s'établir en Occident. Une décennie plus tard, ce chiffre atteignait 40000 personnes (représentant par ailleurs le tiers des personnes les plus qualifiées). De 1985 à 1990, on estime à plus de 60000 le nombre de médecins, d'ingénieurs et de professeurs d'université qui ont émigré de leurs pays. Et aujourd'hui, on pense que cet exode s'est accru, de sorte qu'ils seraient autour de 20000 ceux qui, chaque année, vont vers l'Europe et l'Amérique à la recherche des meilleures opportunités de travail et d'épanouissement.

Pareilles statistiques, comme bien d'autres qui existent au sujet de la fuite des cerveaux, sont rarement dénuées de toute arrière-pensée normative. Sous les apparences d'une simple description sociologique, la notion de fuite des cerveaux cache en elle-même un jugement de valeur sur les devoirs des intellectuels à l'égard de leurs patries. On ne parlerait pas de « fuite » si l'on ne considérait pas tout intellectuel comme étant d'abord rattaché à un pays ou à un continent particulier et ayant à son égard une certaine " obligation patriotique » de contribuer, de toute la force de ses capacités intellectuelles, à son développement, à sa prospérité et à son rayonnement. La fuite des cerveaux s'apparenterait alors à une démission, voire à une trahison de la part de ceux-là même sur qui les États fondaient tous leurs espoirs de mise 
en œuvre des idéaux nationalistes et panafricanistes qui avaient fleuri autour des années des indépendances. Les intellectuels qui ont choisi l'expatriation pourraient être accusés d'avoir voulu accorder la préférence à leurs intérêts particuliers, et de s'être désolidarisés de manière coupable du destin de leurs peuples, de leur continent, d'avoir manqué au devoir d'apporter une contribution, si modeste fût-elle, à la construction de leurs sociétés.

La question pourrait se poser d'emblée de savoir en quoi le patriotisme, le nationalisme et le panafricanisme peuvent être considérés comme des vertus, et si dans la même logique, la fuite des cerveaux pourrait être indexée comme un vice. Le deuxième ordre de préoccupation consisterait à apprécier l'impact réel de la fuite des cerveaux sur le développement et le progrès de l'Afrique. Il faudrait alors pouvoir montrer que seul l'attachement physique d'un cerveau à un lieu déterminé peut lui permettre d'œuvrer pour le compte de ce lieu et qu'il est impossible d'agir sur un lieu à partir d'un autre lieu.

\section{Le patriotisme, le nationalisme et le panafricanisme sont-ils des vertus?}

L'opuscule du philosophe américain Alasdair MacIntyre, intitulé Is Patriotism a Virtue? (1984) pose de façon explicite la question du caractère vertueux du patriotisme. L'auteur y définit le patriotisme comme « une espèce de loyauté à une nation déterminée, que peuvent afficher ceux-là seuls qui possèdent en propre cette nationalité spécifique » (Berten 1998:288). MacIntyre complète sa définition en précisant : « le patriotisme se définit généralement et spécifiquement par une attention particulière portée non seulement à sa propre nation, mais également aux caractéristiques, mérites et exploits de sa propre nation » (280). La loyauté à une nation est ensuite comparée à la fidélité conjugale dans la mesure où le patriotisme requiert un « attachement particulier à une nation » (291), une obligation pour chacun de privilégier au maximum les intérêts de sa communauté.

On peut s'en tenir à cette définition du patriotisme pour comprendre le nationalisme qui, en fait, recouvre globalement la même signification, à quelques nuances près. Être nationaliste, c'est en effet montrer un certain attachement à sa nation, aussi bien sous forme de loyauté préférentielle que sous forme de dévouement pour son devenir. Le panafricanisme correspond à peu près à la même préoccupation : œuvrer pour la libération de la totalité de l'Afrique du joug de la domination étrangère et aussi pour son unité et son développement social, économique et politique. Les historiens nous font savoir que le terme «panafricanisme » apparaît aux Caraïbes vers la fin du XIXe siècle pour désigner un mouvement de révolte des Africains confrontés à l'esclavage en Amérique et à la domination coloniale des Européens en 
Afrique. C'est dans ce contexte que se développe la première version du panafricanisme, qui inscrit, au rang de ses préoccupations les plus urgentes, non seulement l'émancipation des Africains mais aussi, pour certains de ses ténors tels que Edward Blyden et Marcus Garvey, le retour des Noirs vers la terre de leurs ancêtres. Comme l'écrit Nwayila Tshiyembe (2002),

Le rêve panafricain, né sur le continent américain au tournant des XIXe et $\mathrm{XXe}$ siècles, se donnait pour mission de réhabiliter les civilisations africaines, de restaurer la dignité de l'homme noir et de prôner le retour à la «mère patrie »—celle des racines de la diaspora.

Le problème de la valeur morale du patriotisme, du nationalisme, et finalement aussi du panafricanisme réside dans le caractère circonscrit, limité et potentiellement exclusiviste de son champ d'intérêt. En ayant pour visée l'intérêt de l'Afrique et des Africains, on pourrait soupçonner le nationalisme et le panafricanisme d'aller à l'encontre de la morale, si du moins l'on prend l'universalité comme critère essentiel de celle-ci. L'action morale requiert, de ce point de vue cosmopolitique, un arrachement aux contingences et aux intérêts individuels ou communautaires plutôt qu'un attachement à un peuple déterminé et à une histoire particulière. Dans cette conception de la morale, « penser et agir moralement exige de l'agent moral qu'il s'abstraie de toute particularité sociale et de toute partialité » (Berten 1998:291). En privilégiant les intérêts de sa communauté, on pourrait dire que le patriote ou le nationaliste agit de façon intéressée, partiale et que son action est déterminée par des contingences empiriques qui lui ôtent toute moralité.

Certaines figures du nationalisme, comme d'ailleurs certains accents pris par le panafricanisme dans l'histoire, justifient dans une certaine mesure une telle défiance. On rappellera par exemple le nationalisme allemand des années trente, essentiellement xénophobe, qui poussa les autorités allemandes à intervenir dans les pays voisins en alléguant la protection des minorités allemandes prétendument menacées. On peut aussi songer à certaines formes d'afrocentrisme telles celle incarnée aux USA par Leonard Jeffries, Professeur au City University de New York. Ce dernier n'hésitait pas à promouvoir un antisémitisme ouvert (en accusant les Juifs d'avoir financé le commerce des esclaves noirs) et un racisme virulent (en défendant la thèse de la supériorité des Noirs sur les Blancs, traduite par la faible quantité de mélanine chez ces derniers). Le panafricanisme de Marcus Garvey illustre, lui aussi, les dérives possibles d'un attachement trop particulariste aux intérêts d'un peuple déterminé. Dans l'église qu'il avait lui-même fondée, l'African Orthodox Church, les anges étaient peints en noir et le diable en blanc. Il envisagea la création d'une Maison noire comme réplique de la Maison blanche, pour y faire siéger pendant quatre ans celui qui présiderait aux destinées des Noirs 
des États-Unis. Et parmi ses principaux rêves, il y avait le retour de tous les Noirs en Afrique, projet pour lequel il fonda d'ailleurs une compagnie maritime dénommée Black Star Line. Garvey se présentait lui-même comme « le premier des fascistes », et préconisait les méthodes les plus violentes, y compris contre les Noirs qui n'approuvaient pas ses démarches et ses programmes. ${ }^{2}$ On peut ajouter à ces illustrations des dérives du nationalisme l'exemple du kimbanguisme (mouvement religieux indépendant fondé par le catéchiste méthodiste du Congo belge Simon Kimbangu en 1921) pour le raisonnement selon lequel l'Afrique étant le berceau de l'humanité, le peuple africain doit être tenu pour un peuple élu de Dieu ${ }^{3}$ et les Noirs des êtres supérieurs aux Blancs.

Ces exemples pourraient donc justifier que tout nationalisme soit regardé avec une certaine défiance, laquelle s'alimente, comme nous l'avons suggéré plus haut, dans les conceptions universalistes et cosmopolitistes de la morale, dont le kantisme constitue l'une des versions les plus élaborées. Chez Kant, en effet, l'action morale requiert de l'agent moral qu'il s'affranchisse de toute détermination empirique (spatio-temporelle) et passionnelle provenant des intérêts particuliers qu'il veut défendre. Or l'attachement à un pays ou à un continent rétrécit le cercle éthique de l'agent moral pour le ramener à la dimension des intérêts particuliers. Dans le sillage du kantisme, le philosophe australien Peter Singer englobe dans la même objection le racisme, le nationalisme, le patriotisme et même l'amitié. Les points importants de sa thèse sont résumés par Bernard Baertschi :

Les racistes violent le principe d'égalité en donnant un plus grand poids aux intérêts des membres de leur propre race quand ils entrent en conflit avec les intérêts des membres d'une autre race.

Les nationalistes violent le principe d'égalité en donnant un plus grand poids aux intérêts des membres de leur propre nation quand ils entrent en conflit avec les intérêts des membres d'une autre nation.

Les patriotes violent le principe d'égalité en donnant un plus grand poids aux intérêts des membres de leur propre patrie quand ils entrent en conflit avec les intérêts des membres d'une autre patrie (Baertschi 2002:62).

À la lumière de ces critiques universalistes et libérales du nationalisme et du patriotisme, on pourrait affirmer qu'aucun individu n'a à considérer l'attachement à son peuple comme un devoir. Au contraire, pareil attachement serait une violation de l'interdit moral de privilégier les intérêts de son milieu. Par conséquent, le choix pour un intellectuel de s'installer sous d'autres cieux ne tombe pas dans les rets d'une critique morale de la fuite des cerveaux, 
à moins que ce choix soit déterminé par la seule motivation de la maximisation de ses intérêts égocentriques. Comme disait Cicéron, citant Pacuvius : « Ma patrie, c'est là où je suis bien », ou plus récemment H. G. Wells : « Notre véritable nationalité est l'humanité » (Miller 2002:32). Non seulement l'intellectuel qui émigre exerce son droit à la mobilité géographique et sociale, mais encore il met ses compétences au service de l'humanité, plutôt que de les laisser s'émousser dans sa propre communauté. ${ }^{4} \mathrm{C}$ 'est par ce désintérêt pour les préoccupations nationalistes et panafricanistes qu'il exprimerait son impartialité et son attachement à des valeurs universelles.

À cette conception libérale de la morale et du patriotisme, fondée sur le refus des préférences particularistes, s'oppose celle qui fait des particularités sociales, communautaires et culturelles, le cadre de référence qui donne sens et contenu à l'identité humaine et à sa moralité. Comme dit MacIntyre (Berten 1998:297), « d'une manière générale, ce n'est qu'au sein d'une communauté que les individus deviennent capables de moralité et sont soutenus dans leur moralité ». Il n'existe pas de morale universelle, détachée de tout enracinement communautaire. L'homme étant par nature, selon les mots d'Aristote, un « animal politique », celui qui se définit indépendamment d'une communauté peut être assimilé à un monstre ou à un être dégradé. Ce statut de sujet politique ou communautaire implique un devoir d'allégeance à la communauté à laquelle on appartient, la communauté qui précisément nous définit comme sujet politique, et même comme sujet tout court. La première des vertus pourrait donc être cette allégeance elle-même, toutes les autres vertus ne pouvant être accomplies qu'à l'intérieur du cercle éthique circonscrit par notre communauté.

L'attachement à ma communauté, à la hiérarchie d'une structure de parenté définie, d'une communauté locale particulière et d'une communauté naturelle déterminée est, de ce point de vue, un prérequis à toute morale. Ainsi, le patriotisme et toutes les loyautés analogues ne sont plus seulement des vertus, mais passent au rang de vertus fondamentales (Berten 1998:298).

Bernard Baertschi parle du « charme secret du patriotisme », à travers une défense de ce qu'il appelle le «principe de proximité morale ». Selon ce principe, il n'est point illégitime d'avoir une certaine préférence pour ceux qui nous sont proches. Or, en général, c'est la communauté à laquelle on appartient qui crée les conditions premières de la proximité : d'abord factuelle, cette proximité crée les relations causales (d'interaction réciproque) et symboliques sur lesquelles peut aussi se fonder une proximité culturelle, religieuse, morale, etc. La proximité morale fonde le raisonnement moral selon lequel si tel individu est plus proche de moi que tel autre, ses intérêts importent plus pour moi que ceux du second. 
Ce raisonnement s'expose sans doute à l'objection de paralogisme naturaliste. Car d'une déduction factuelle, on passe à une déduction normative du type "les intérêts de tel individu proche de moi doivent beaucoup plus compter pour moi que ceux des autres". Mais l'objection n'est valable que si l'on suppose que les normes éthiques ne doivent pas s'enraciner dans la réalité humaine elle-même. Or, comme tente de l'expliquer Bernard Baertschi il s'agit simplement du « fondement anthropologique de l'éthique normative, et sous la réserve qu'il en existe une justification éthique » (Berten 1998:6970). Par notre appartenance à une communauté (réalité anthropologique), nous partageons la même histoire, mais aussi les valeurs de compassion, d'amitié, de solidarité, etc. C'est cette proximité qui circonscrit le cercle éthique à l'intérieur duquel nous avons des devoirs, même si ce cercle ne doit pas être considéré comme étant fermé à une perspective universelle. Faire de la communauté le cercle éthique prioritaire ne consiste pas à poser une communauté contre d'autres communautés, mais simplement à montrer d'un point de vue anthropologique qu'une perspective universaliste risque de se réduire à une pure abstraction. C'est toujours d'abord à l'égard des personnes appartenant à notre communauté que nous avons des devoirs moraux, à moins que nous ne préférions nous tourner vers la personne humaine en général, que, dans un certain sens et pour reprendre l'idée de Joseph de Maistre, nous ne rencontrons jamais.

Ce n'est jamais envers des personnes en général, ni même envers des concitoyens abstraits que nous avons des responsabilités morales - sources de devoirs -, mais face à des individus, Pierre ou Paul, qui sont des personnes et des concitoyens, c'est-à-dire des êtres qui, dès l'abord, sont membres de diverses communautés avec lesquelles nous entretenons des rapports de proximité, larges ou serrées (Berten 1998:78-79).

Il n'y a là aucune contradiction avec les principes d'impartialité et de justice, qui, en fait, ne supposent pas nécessairement un cercle éthique élargi à l'humanité entière.

Afin d'éviter que les deux morales opposées - morale universaliste et morale communautariste- ne nous enferment dans un dilemme, il est heureusement possible de les concilier de façon féconde en disant que le patriotisme ou le nationalisme signifie simplement l'allégeance au projet de constitution d'une communauté historique, lequel projet se réalise par des actions et des événements déterminés. Être patriote, c'est partager les efforts grâce auxquels une nation ou une communauté se crée, se constitue et se réalise au fil de l'histoire. Et dans la mesure où seul le projet justifie l'allégeance patriotique, et pas nécessairement la simple affinité raciale ou ethnique, il est tout aussi patriotique de s'opposer à certaines formes que 
peut prendre la réalisation de ce projet et qui sont susceptibles d'en dévier la trajectoire idéale. Que cette allégeance soit tournée électivement vers la promotion d'une communauté particulière et non de l'humanité en général ne lui enlève pas son importance et même sa valeur morale. L'humanité est en réalité organisée en communautés particulières appelées chacune à assurer sa survie en tant que communauté. Cette survie dépend de l'allégeance et du dévouement de chaque individu qui, par sa contribution, réalise sa propre survie, celle de sa communauté et celle de l'humanité en tant qu'ensemble de communautés.

Donc, bien qu'il y ait tout lieu de craindre que le patriotisme, le nationalisme et le panafricanisme soient immoraux, en ce que la maxime qui les détermine est fondée sur des réalités particulières, voire sur des intérêts partisans, ces réalités et ces intérêts ne sont pas nécessairement contraires à la promotion de l'humanité qui ne se présente pas autrement que comme une « communauté de communautés ».

\section{La « fuite » des cerveaux et le devoir de contribuer à la construction de l'Afrique}

Rousseau disait des cosmopolites qu'ils « se vantent d'aimer tout le monde pour avoir le droit de n'aimer personne » (Baertschi 2002:75). Et avant Rousseau, Platon dans Le Criton faisait voir à travers l'acceptation par Socrate de la sentence injuste du tribunal d'Athènes qui le condamnait à boire la ciguë combien était important l'attachement d'une personne à sa patrie. À son ami Criton qui lui offrait l'opportunité de s'évader pour échapper à cette mort injuste, Socrate répondit par une prosopopée des lois :

N'est-ce pas à nous que tu dois la vie et n'est-ce pas sous nos auspices que ton père a épousé ta mère et t'a engendré ? Parle donc : as-tu quelque chose à redire à celles d'entre nous qui règlent les mariages ? Les trouves-tu mauvaises ? [...] Et à celles qui président à l'élevage de l'enfant et à son éducation, éducation que tu as reçue comme les autres? Avaient-elles tort celles de nous qui en sont chargées, de prescrire à ton père de t'instruire dans la musique et la gymnastique ? [...] Après que tu es né, que tu as été élevé, que tu as été instruit, oserais-tu soutenir d'abord que tu n'es pas notre enfant et notre esclave, toi et tes ascendants ? [...] Qu'est-ce donc que ta sagesse, si tu ne sais pas que la patrie est plus précieuse, plus respectable, plus sacrée qu'une mère, qu'un père et que tous les ancêtres, et qu'elle tient un plus haut rang chez les dieux et chez les hommes sensés... ? (Le Criton 50c-51b).

L'évasion de Socrate, selon cette prosopopée, aurait paru comme un acte d'ingratitude sacrilège : « La patrie est plus précieuse, plus respectable, plus 
sacrée qu'une mère, qu'un père... ». La cité fournit à chacun tout ce qu'il y a de plus important pour une vie humaine accomplie : la vie et l'éducation. Elle est donc en droit d'attendre en retour une allégeance à ses institutions, à la fois sous la forme d'une obéissance inconditionnelle à ses lois et d'une contribution à l'amélioration de celles qui sont défectueuses. La prosopopée admet en effet la possibilité d'un changement des règles considérées comme mauvaises, afin que, par ces améliorations, les lois s'accordent toujours avec l'idéal de bien qu'elles sont censées traduire. Point n'est besoin, pour ce faire, de prendre le chemin de la révolution, moins encore de l'exil : « Il faut faire ce qu'ordonnent l'État et la patrie, sinon la faire changer d'idée par des moyens qu'autorise la loi » (Le Criton 51b).

Sur le modèle de la prosopopée des lois, l'intellectuel émigré pourrait entendre la complainte suivante :

Nous avons consenti pour toi d'énormes sacrifices en construisant des écoles et des universités pour assurer ta formation et des hôpitaux pour veiller sur ta santé. Comment peux-tu, une fois ta formation achevée, quitter ta patrie sans te soucier de ce que deviendront toutes ces institutions qui t'ont tout donné ? Serais-tu heureux d'apprendre que les écoles, les hôpitaux, les usines ont disparu faute de personnes qualifiées pour les faire fonctionner? Accepteras-tu que ton pays dépérisse parce que ceux qui étaient censés le développer ont choisi d'assurer leur bien-être particulier ailleurs ?

Il y aurait dans le subconscient de chaque intellectuel une sorte de « démon » prêt à lui adresser de telles remontrances au cas où il voudrait se dérober de l'obligation de contribuer à l'édification de l'Afrique. Ce démon aurait pour mission « sacrée » de rappeler en quelque sorte l'éthique patriotique contraire à l'attitude des free riders enclins à maximiser leurs intérêts par la recherche d'emplois fortement lucratifs sous d'autres cieux (Van Parijs 1993:309-342). Il serait difficile pour l'intellectuel dont les prouesses sont reconnues et vantées dans un pays différent du sien que la gratification qu'il pourrait légitimement tirer de cette reconnaissance ne soit pas au moins tempérée par la frustration de ne pas pouvoir faire bénéficier directement cette ingéniosité à sa propre communauté. De l'étranger où il excelle, il ne peut être indifférent à l'histoire de sa communauté, à ses succès qui le comblent de fierté, mais aussi à ses échecs qui le remplissent de honte et de regret. C'est pourquoi l'on peut raisonnablement soutenir, avec David Miller, que « la communauté nationale historique est une communauté d'obligation. Parce que nos prédécesseurs ont travaillé dur et versé leur sang pour construire et défendre la nation, nous qui y sommes nés, héritons l'obligation de continuer leur travail, dont nous nous déchargeons en partie sur nos contemporains et en partie sur nos descendants »(2002:36). Les nations seraient vouées à la 
disparition si leurs membres ne faisaient pas de cette tâche de participer à leur construction une véritable obligation morale et patriotique. Il faudrait, pour ceux qui partent, que les raisons de partir ne soient pas antipatriotiques et que le geste même de partir, le geste de se détacher, puisse être déterminé et justifié en même temps par un certain attachement à sa patrie.

Chez MacIntyre qui attache comme on l'a vu beaucoup de prix à l'allégeance d'un individu à sa communauté, cette allégeance est conditionnelle : on ne peut en effet attendre des individus l'allégeance à une communauté politique dans laquelle la notion de bien commun n'a aucune signification et qui s'apparente à un casino où chaque joueur s'efforce de maximiser son gain.

Une communauté nationale qui renierait systématiquement sa propre histoire véritable, une communauté nationale dans laquelle les liens tissés par l'histoire ne seraient pas les liens proprement fédérateurs de la communauté (ceux-ci ayant par exemple été remplacés par des liens d'intérêt mutuel), serait une communauté à l'égard de laquelle le déploiement d'une attitude patriotique s'assimilerait, à tout point de vue, à une attitude irrationnelle. (Berten 1998:305)

L'attachement à une patrie n'est raisonnable, du point de vue suggéré ici par MacIntyre, que si cette patrie développe le sens de l'appartenance commune, à travers des politiques et des institutions dont le fonctionnement exprime sans ambiguïté l'idéal de solidarité et de bien commun.

Or, les États africains, depuis les indépendances jusqu'à nos jours, ont rarement présenté le visage de véritables communautés politiques, dans le sens que les anciens donnaient à cette notion de communauté. Chacun de ces États peut certes faire prévaloir une certaine unité symbolisée par un nom (le Cameroun, le Tchad, le Gabon, par exemple), un drapeau, un territoire (aux contours plus ou moins bien définis dans certains cas), un pouvoir (plus ou moins stabilisé), etc., mais il ne s'agit dans la plupart de cas que d'une unité formelle, artificielle, qui est au fond minée par des clivages identitaires entretenus par des entrepreneurs politiques en mal de conquête ou de conservation du pouvoir. L'histoire de ces sociétés se tisse souvent autour de la problématique du pouvoir à conserver ou à conquérir, ce qui les réduit à des espaces d'affrontements feutrés ou ouverts. Chaque acteur s'efforce, au mieux de son ingéniosité, à tirer son épingle du jeu, en tâchant soit d'obtenir le maximum d'avantages possibles, soit de résister, simplement, au risque permanent de disparition.

Les sociétés africaines qui sont concernées par la fuite des cerveaux, en tant que problème, par rapport à leur besoin de l'expertise des intellectuels sont en général celles qui, dès la période des indépendances, ont adopté à l'égard de la classe des intellectuels trois types d'attitudes: 
1. l'intimidation, pour ceux qui, déployant leur sens de la lucidité critique, ont tenté de mettre à nu les défaillances, les ruses, la mauvaise foi, le cynisme et les fuites en avant des détenteurs du pouvoir. C'est ce qui explique le fait qu'un nombre important d'intellectuels émigrés soient des exilés politiques.

2. La stérilisation du génie intellectuel par son asservissement à l'idéologie et aux pratiques sordides des régimes politique en place.

3. La précarisation et la clochardisation des intellectuels, comme au Cameroun où, en moyenne, les salaires des agents de l'armée, de la police, de la gendarmerie, de la justice et de tout ce qui constitue ce que Louis Althusser a appelé « appareils répressifs d'État », de même que ceux des journalistes des médias d'État (« appareils idéologiques d'État») ont pu atteindre autour des années 1994 le double, voire le triple des salaires des médecins, des chercheurs, d'enseignants d'universités, etc.

La fuite des cerveaux, dans ces conditions, peut être considérée comme la réponse la plus appropriée, la plus vertueuse, à l'obligation pour chaque intellectuel de valoriser les dons qu'il possède. Et on devrait pouvoir y voir une forme de patriotisme qui n'est plus allégeance directe et physique à sa communauté, mais à l'humanité à travers une autre communauté qui rend possible la valorisation du génie intellectuel. Quand le principe régulateur de l'existence dans une société se réduit au struggle for life, il appartient à chacun de trouver les meilleures stratégies de sa survie. Et quand, parallèlement à l'impossibilité d'assurer cette survie à l'intérieur, se profile l'opportunité de le faire ailleurs, il est rationnel, voire raisonnable, de recourir à cette opportunité.

On pourrait voir dans ce départ une certaine lâcheté, l'attitude la plus vertueuse devant consister à affronter le danger de l'intérieur. ${ }^{5}$ Fallait-il par exemple qu'Aristote quittât Athènes pour éviter, selon sa propre justification, qu'un autre crime ne soit commis contre la philosophie, après celui qui avait été perpétré contre Socrate ? En acceptant de mourir suite à une condamnation et à un procès injustes, Socrate disait que les raisons de mourir valent parfois mieux que les raisons de vivre. Mais pour ce qui concerne les cerveaux, un cerveau vivant, même loin du lieu où on souhaite qu'il se trouve, vaut toujours mieux qu'un cerveau mort dans son propre terroir.

Cette allégeance à l'humanité à travers d'autres communautés constitue, dans bien des cas heureusement, un autre moyen efficace pour les intellectuels de contribuer au développement de leur propre communauté. Il est en effet loisible de voir, à travers de nombreux exemples, que la mise en valeur de compétences des intellectuels africains, sous d'autres cieux, a énormément contribué à l'essor de l'Afrique elle-même, sur le triple plan culturel, économique et politique. Sur le plan culturel, les grands idéaux du 
panafricanisme et de l'indépendance doivent leur impulsion aux Africains de la diaspora. C'est bien aux Caraïbes, aux États-Unis et au Brésil que le panafricanisme a vu le jour, bien avant que ne s'y associent, depuis l'Afrique, des intellectuels et hommes politiques comme Kwame Nkrumah, Kenneth Kaunda, Julius Nyerere, Jomo Kenyatta, Haïlé sélassié, Namdi Azikiwe, Peter Abrahams, etc. Confrontés à la discrimination et au mépris, ces Africains ont pu développer un sens très aigu de leur appartenance à une culture déterminée, et initier des démarches pour la reconnaissance et la mise en valeur de cette culture. Le combat pour l'abolition de l'esclavage, auquel nombre d'entre eux étaient plus directement confrontés, ne pouvait pas ne pas s'accompagner d'un combat pour la libération de l'Afrique. On peut ici évoquer la conférence panafricaine organisée à Londres en 1900, sous la houlette du Trinidadien Henry Sylvester Williams et le Haïtien Benito Sylvain, à partir d'une organisation, la pan-african association, qu'ils avaient mise sur pied dès 1897. On peut ajouter 1'action de Olaudah Equiano (Gustavo Vassa), esclave affranchi installé en Grande Bretagne, qui, au XVIIIe siècle, avait parcouru tout le Royaume-Uni pour mener une campagne contre l'esclavage et plaider pour son abolition. Le congrès panafricain des militants africains, organisé à Manchester en 1941, joua un rôle historique de premier plan dans le processus de la décolonisation. C'est sous la houlette d'un autre Trinidadien, George Padmore, que s'organisa également à Manchester le $5^{\mathrm{e}}$ congrès panafricain, lequel adopta un manifeste formulant l'engagement suivant : « Nous sommes résolus à être libres... Peuples colonisés et assujettis du monde, unissez-vous. » On pensera aussi au rôle des intellectuels africains présents en France, comme Léopold Sédar Senghor, Alioune Diop, autour des revues comme Présence africaine, sans compter le mouvement de la Négritude qui a joué (et joue encore) un rôle ô combien important dans la formation des jeunes Africains.

Sur le plan scientifique, il y a, comme l'écrivent Anne-Marie Gaillard et Jacques Gaillard, des pays « qui finissent par gagner » (2002) dans cet exode des cerveaux. Certaines études ont certes pu établir le coût du manque à gagner dans les pays dont une bonne proportion des plus qualifiés s'expatrie (Sethi 2000:39). Mais le brain drain peut se transformer en brain gain si ceux qui partent veulent contribuer au développement scientifique de leurs terroirs. On en a quelques exemples en Asie, Afrique et Amérique latine, où des pôles de collaboration scientifique et des réseaux de connexion avec les expatriés ont été mis en place. ${ }^{6}$ "Ces réseaux cherchent d'avantage à canaliser la science et la technologie plutôt que les hommes et cherchent également à initier des coopérations avec les pays développés accueillant leurs élites »(Gaillard 2002). 
L'apport économique de la diaspora africaine n'est pas moins remarquable. Le président ougandais, Yuweri Museveni n'hésite pas à considérer les Ougandais de l'extérieur comme étant « l'exportation la plus importante du pays ». Leur apport se chiffre à 400 millions de dollars par an, montant largement supérieur à ce que rapporte le café qui est pourtant le premier produit agricole du pays. On pourrait trouver des chiffres similaires dans d'autres pays, comme par exemple au Ghana, où l'apport des nationaux installés à l'étranger se situe entre 350 et 450 millions de dollars par an. Des travaux sur la contribution des travailleurs maliens et sénégalais résidents en France révèlent la même importance de la diaspora. Parlant du cas des Maliens, Libercier et Hartmut (1996) écrivent :

Sur une dizaine d'années, ils ont financé 146 projets représentant un budget total de 19,4 millions de francs français, dont 16,6 millions de francs étaient financés sur leurs économies, les 2,8 millions restants ayant été offerts par des ONG avec l'aide des donateurs internationaux. C'est ainsi que la réalisation de $64 \%$ de l'infrastructure des villages de la région de Kayes est attribuée aux travailleurs migrants.

L'apport économique des Africains de la diaspora se conjugue donc à leur apport culturel et scientifique pour montrer que s'il y a eu «fuite» des cerveaux, cette fuite est bien loin d'avoir causé l'échec-si échec il y a $\mathrm{eu}$ - des grands idéaux du nationalisme et du panafricanisme. Au contraire, loin que la «fuite» ait (toujours) été dommageable pour l'Afrique, elle a contribué à l'aider à penser la question de son identité, à édifier ce qu'elle a de meilleur, comme aussi peut-être ce qu'elle a de pire. Si la contribution des Africains de la diaspora au développement du continent (qui, d'après certaines données, est supérieure à l'aide internationale au développement) n'a pas réussi à sortir l'Afrique de sa marginalité et de sa misère de plus en plus grave, il faut maintenant s'interroger sur les facteurs qui entretiennent cette déchéance. Et peut-être la présence de " cerveaux » africains dans ces lieux où se joue une bonne partie du sort de l'Afrique peut-elle aider à éviter que lui soient toujours réservés les mauvais lots.

\section{Conclusion}

Les deux préoccupations qui auront retenu notre attention dans cette réflexion nous conduisent à penser que la notion de fuite des cerveaux doit être délestée de la charge culpabilisante qui l'a toujours accompagnée. Si on la considère comme l'expression d'un manquement à l'obligation patriotique de se dévouer pour sa communauté, il faudrait encore que le patriotisme soit considéré d'emblée comme une vertu. Or, cette position ne va pas sans dire. Et même quand on ne partage pas absolument l'approche cosmopolite de la question 
(selon laquelle le particularisme qui caractérise essentiellement l'attachement patriotique est contraire à la morale), on doit pouvoir admettre que ce qui donne sens au patriotisme, ce n'est pas la pure fidélité à une communauté, mais l'allégeance à un projet de constitution de bien commun. Il faut donc d'abord présupposer l'existence de pareil projet pour que le choix de l'exode soit considéré comme une « fuite », une dérobade par rapport à l'obligation d'apporter sa contribution au développement de ce projet. Dans le contexte africain, il y a eu des projets visant la promotion de l'Afrique en tant que communauté unique de destin. Et si ces projets n'ont pas été mis en œuvre, comme ils avaient été pensés au départ, ce n'est pas aux intellectuels émigrés qu'on pourrait en imputer la responsabilité. Car en fin de compte, ce n'est pas au niveau de l'exode géographique des cerveaux qu'il faudrait situer la « fuite », comme on le fait toujours, mais au niveau d'une évasion vers des préoccupations n'ayant aucun rapport avec ce dont l'Afrique a besoin pour se libérer et se développer. Et dans ce cas, la notion pourrait pertinemment s'appliquer à la débauche des cerveaux qu'on observe chez ceux qui restent et qui préfèrent s'aliéner aux régimes tyranniques qui leur garantissent leurs intérêts égoïstes plutôt que de se mettre au service de leur peuple. En somme, la fuite des cerveaux ne se trouve pas toujours là où on croit la voir.

\section{Notes}

1. Garvey taxait par exemple Dubois d'« ennemi déclaré de la race noire » et de «mulâtre paresseux et vendu ». Cf. Decraene,Ph., 1959, Le panafricanisme, $\mathrm{n}^{\circ} 847$ de la collection « Que sais-je », Paris: PUF, p. 18.

2. On trouve un schéma quasi identique dans l'Église hollandaise réformée, en Afrique du Sud de la période de l'Apartheid, qui, fournissant les fondements religieux du système de ségrégation raciale, affirmait que les Afrikaners étaient le peuple élu de Dieu et les Noirs une espèce subordonnée. Cf. Mandela, N., 1995, Un long chemin vers la liberté, Fayard, p. 138.

3. De nombreuses études ont en effet montré que, pour certains pays au moins, le départ des intellectuels est dû en partie à l'impossibilité pour ces pays de les absorber dans leur marché de l'emploi.

4. C'est l'une des objections contenues dans le commentaire critique de ce texte par Nana Akua Anyidoho de la Northwestern University aux USA. Je la remercie pour la pertinence de ce commentaire qui a attiré mon attention sur certaines ambiguïtés de mes arguments.

5. Voir l'étude assez détaillée consacrée aux dispositifs mis en place par les Africains de la diaspora pour contribuer au développement de la science dans leurs pays d'origine par Mercy Brown, « Using Intellectual Diaspora to Reverse the Brain Drain », ECA/IDRC/IOM (éds.), Brain Drain and Capacity Building in Africa, ECA/IDRC/IOM, 2000, pp. 92-106. 


\section{Références}

Baertschi, B., 2002, « Les charmes secrets du patriotisme », in Les nationalismes, Bernard Baertschi et Kevin Mulligan Éds., Paris: PUF.

Berten, A. et al., 1998, Libéraux et communautariens, Paris: PUF.

Brown, M., 2000, « Using Intellectual Diaspora to reverse the Brain Drain », in Brain Drain and Capacity Building in Africa, ECA/IDRC/IOM.

Decraene,Ph., 1959, Le panafricanisme, $\mathrm{n}^{\circ} 847$ de la collection « Que sais-je », Paris: PUF.

ECA/IDRC/IOM, éds., 2000, Brain Drain and Capacity Building in Africa, (bilingual edition: Exode des compétences et développement des capacités en Afrique), ECA/IDRC/IOM.

Gaillard, A.M., Gaillard, J., 2002, « Fuite des cerveaux, circulation des compétences et développement : un enjeu politique », in Mots pluriels, $\mathrm{n}^{\circ} 20$ (http:// www.arts.uwa.edu.au/MotsPluriels/MP2002ajg.html).

Libercier, M., Schneider, H., 1996, Migrants: Partners in Development Cooperation, Paris: OECD.

MacIntyre, A., 1984, Is Patriotism a Virtue?, The Lindley Lecture, University of Kansas (Traduction in Berten, A. et al., 1998, Libéraux et communautariens, Paris: PUF.

Mandela, N., 1995, Un long chemin vers la liberté, Paris: Fayard.

Miller, D., 2002, « Une défense de la nationalité », in Les nationalismes, Bernard Baertschi et Kevin Mulligan Éds., Paris: PUF.

Platon, Le Criton.

Sethi, M., 2000, 'Return and Integration of Qualified African Nationals', in Brain Drain and Capacity Building in Africa, ECA/IDRC/IOM.

Tshiyembe, M., 2002, « Du messianisme aux rives de la mondialisation. Difficile gestation de l'Union africaine », Le Monde diplomatique.

Van Parijs, P., 1993, 'Rawlsians, Christians and Patriots: Maximin Justice and Individual Ethics', European Journal of Philosophy, Vol. 1, no. 3. 